\title{
ULTRASOUND USE OF POST-TRAUMATIC GLUTEAL HAEMATOMA IN A PATIENT USING WARFARIN
}

\author{
Fatih Demir $^{1} \odot$, Gülden Kazancı ${ }^{2}$, Jacek Smereka ${ }^{3}$, Damian Gorczyca ${ }^{4} \odot$ \\ ${ }^{1}$ Department of Emergency Medicine, Ufuk University Medical Faculty, Dr Ridvan Ege Education and Research Hospital, Ankara, Turkey \\ ${ }^{2}$ Department of Emergency Medicine, Ankara Training and Research Hospital, Ankara, Turkey \\ ${ }^{3}$ Department of Emergency Medicine, Wroclaw Medical University, Wroclaw, Poland \\ ${ }^{4}$ Department of Emergency Medicine, Lazarski University, Warsaw, Poland
}

\begin{abstract}
Most of the muscle pathologies consist of traumas. The use of oral anticoagulants increases post-traumatic bleeding and haematoma risk. Ultrasonography has an important place in diagnostic examination of the soft tissue haematomas that appear in post-traumatic period. In this study a patient is presented who used warfarin and was admitted at the emergency department with hip pain and difficulty in walking, and who had gluteal haematoma on ultrasound after falling down. Hip pains in patients using warfarin must be alarming in terms of gluteal haematoma. Ultrasound offers fast and reliable results in preventing complications.
\end{abstract}

KEY WORDS: haematoma; trauma; gluteal muscle; warfarin; ultrasound

Disaster Emerg Med J 2019; 4(3): 124-127

\section{INTRODUCTION}

The reasons for a haematoma in the gluteal region can be listed as trauma, anticoagulant use, disorders in coagulation, and iatrogenic injuries [1-6]. Patients present to healthcare institutions with pain and discolouration of the skin in this area. There is also a risk of developing gluteal compartment syndrome due to massive haematomas [7]. Myositis ossification complications may also develop in the chronic period [8]. There are three compartments in the gluteal area. There is a risk of compressing the sciatic nerve due to haematomas that occur in the compartment formed by the gluteus maximus muscle [1]. Drainage of the haematoma in early period reduces the risk of complications. The use of anticoagulant causes spontaneous haematoma, and affects the width of the post-traumatic haematoma, and increases the risk of complication by prolonging the duration of haemorrhage. The increase in the volume of muscle tissue, oedema, fluid accumulation, haematoma, muscle ruptures, and infection findings can be detected with ultrasonography [9]. The patient who had post-traumatic haematoma in the gluteal area has been presented in this case study to emphasize the importance of ultrasound imaging in diagnosing and following-up the haematoma.

\section{CASE REPORT}

A 72-year-old male patient presented to the Emergency Department with pain in the right hip and leg. It was learned from the history of the patient that he had applied to the Emergency Department due to a pain starting on his right hip as a result of falling down from the same level five days ago; and due to bruising that spread to his ankle from the waist area. The patient presented to another healthcare centre on the first day of the falling down and was discharged upon the detection of no acute pathologies. In the history of the patient, 
he was followed with chronic obstructive pulmonary disease, hypertension and diabetes. It was reported that the patient had undergone mitral valve replacement 15 years ago, and due to this, he was using coumadin. In the physical examination of the patient, it was determined that the general condition was good. Temperature: 36.7 , TA: $110 / 70 \mathrm{~mm} \mathrm{Hg}$, $\mathrm{SO}_{2}$ : 95, Pulse: 85. Ecchymosis was detected that started from the right lumbar area extending to the base of the right foot (Fig. 1). No joint limitations were detected in the hip movements of the patient. Tenderness was detected with palpation in the right gluteal area. Homan's sign for deep venous thrombosis was negative. There were no motor or sensory deficits in the right lower extremity of the patient. The right hip radiograph of the patient was taken, and the patient was consulted with the orthopaedic department. No acute pathologies were detected in the radiograph. Complete blood count, renal function tests and bleeding parameters were examined.

The following measurements were made in the patient: WBC: 8561 cells/mcL, HGB: 9.94 gr / dL, PLT: 282000/mcL, BUN: 17, Creatinine: 0.79, CRP: 21.0, INR: 2.62. A control haemogram was carried out at the $12^{\text {th }}$ hour for the patient who had low haemoglobin level. HGB was measured as $9.45 \mathrm{mg} / \mathrm{dl}$. The patient underwent ultrasound at the admission to the Emergency Department (Fig. 2), and at the $12^{\text {th }}$ (Fig. 3), 24 ${ }^{\text {th }}$ (Fig. 4) and $48^{\text {th }}$ (Fig.5) hours. The haematoma area in the gluteal region of the patient was visualized by using Logiq @ 200 brand $7.5 \mathrm{MHz}$ linear probe. The hyperechogenic areas that were compatible with the fibrin accumulations in the haematoma radiograph were consistent with the five-day history of the patient. The hypoechoic areas that were considered to be the focus of haematoma area were visualized (Fig. 1). As the patient had undergone mitral valve replacement, the patient was consulted with cardiology and cardiovascular surgery departments. Their recommendations were received for warfarin use. Because of the presence of fibrin accumulations detected in bedside ultrasound imaging (POCUS), the detection of no expansion in the haematoma area in the ultrasonographic follow-ups, and the haemodynamics of the patient being stable, the patient was not considered to be given cofactor or fresh frozen plasma. As the bedside serial ultrasound findings were compatible with the current clinic of the patient, CT angiography was not considered to be suitable for radiation safety, and clinical follow-ups were made for the patient. He

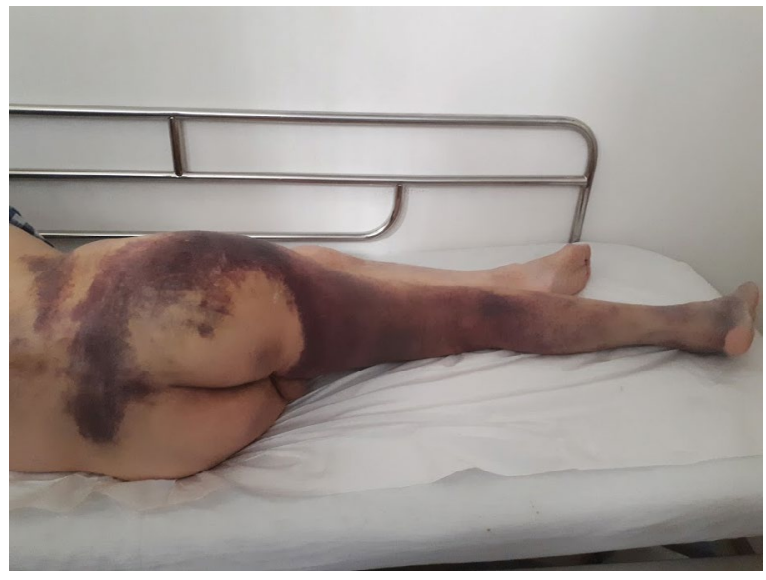

FIGURE 1. The ecchymosis area stretching from the lumbar area of the patient to the sole of the right feet

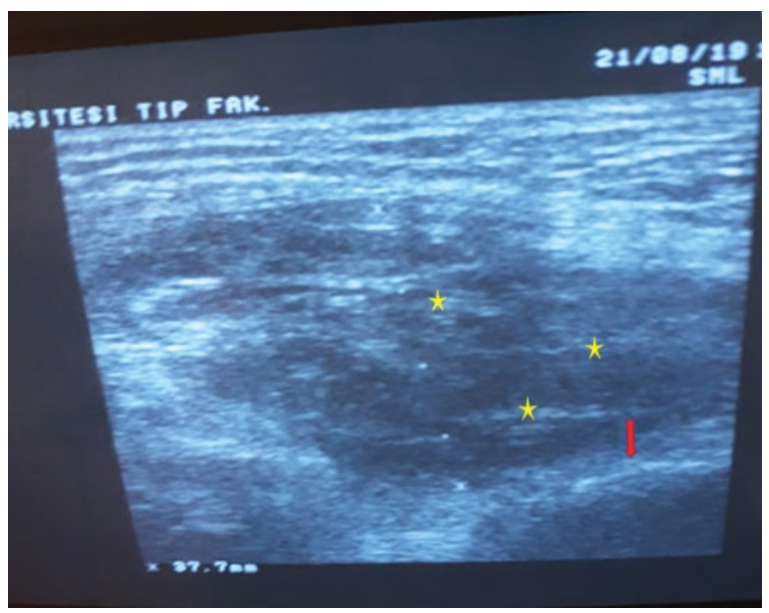

FIGURE 2. Transverse scan. The initial ultrasound ( $5^{\text {th }}$ day after trauma). Transverse diameter $37.7 \mathrm{~mm}$. Areas with star show the fibrin accumulation and Coxa is seen in the tip of the red arrow on haematoma

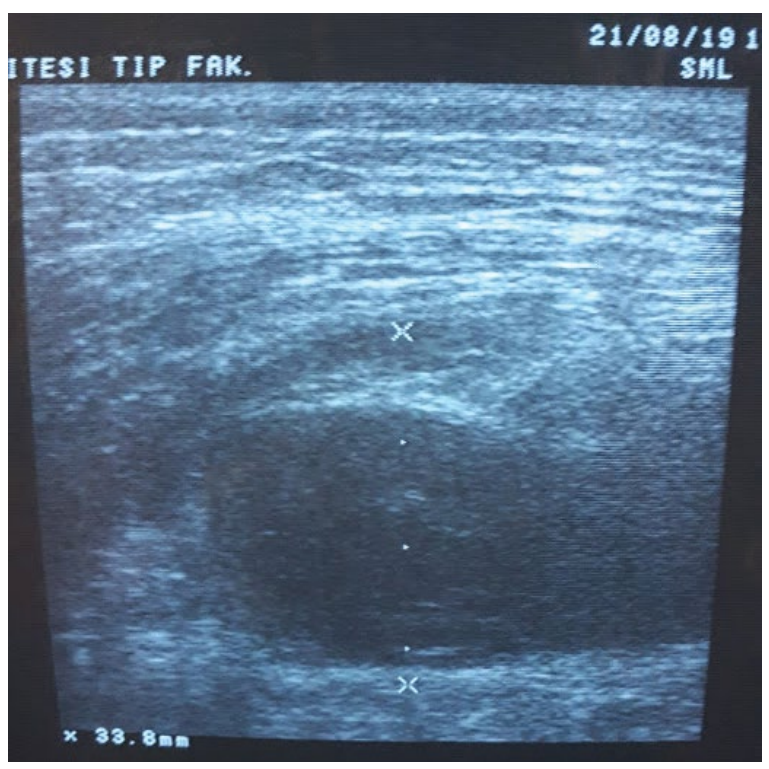

FIGURE 3. Transverse scan at the $12^{\text {th }}$ hour control ultrasound. Transverse diameter: $33.8 \mathrm{~mm}$ 


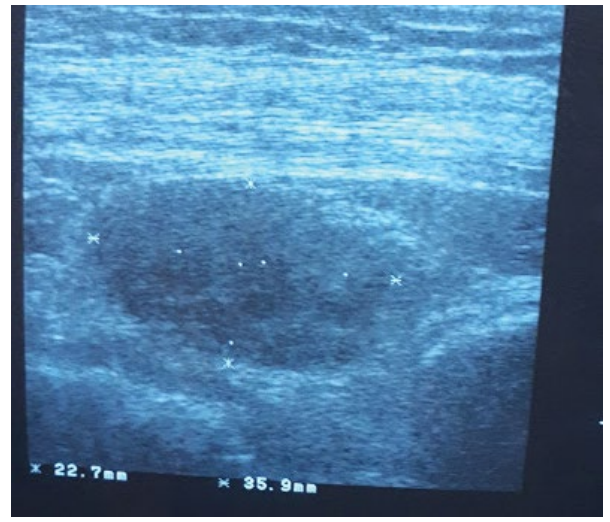

FIGURE 4. Transverse scan. $24^{\text {th }}$ hour control ultrasound. Transverse diameter: $22.7 \mathrm{~mm}$

was followed up for compartment syndrome. The orthopaedic department recommended cold application and foot elevation. There were no changes in the patient in terms of haemogram, blood pressure and pulse follow-ups at the $12^{\text {th }}$ and $24^{\text {th }}$ hours. The patient was discharged when his haemodynamics was detected to be normal, and there was no decrease in haemoglobin at the $48^{\text {th }}$ hour (Fig. 5).

\section{DISCUSSION}

The development of haematoma in muscle and soft tissue after trauma is among the expected conditions [10]. There are several case reports in the literature conducted on development of spontaneous haematoma in patients who take coumadin [11]. Although the use of new-generation oral anticoagulants like rivaroxaban is increasing, studies on their toxic effects are limited in the literature [12]. There were no studies conducted on soft tissue haematoma after traumas in patients who receive anticoagulants. In a study published by Liu et al. in 2019, a total of 30 patients who had closed extremity fractures were evaluated with ultrasound; and it was reported that $6(20 \%)$ of them had haematoma that was $4 \mathrm{~cm}$ in diameter [13]. In another case report, gluteal haematoma following deep tissue massage therapy was reported [14]. In our patient, complications that might develop can be listed as the continuation of the bleeding, compartment syndrome, infection, and long-term myositis ossificans. Post-traumatic myositis ossificans is a more common complication in this respect [15]. Muscle haematoma develops due to ossification. In the literature, there is a case report on gluteal haematoma caused by superior gluteal artery injury

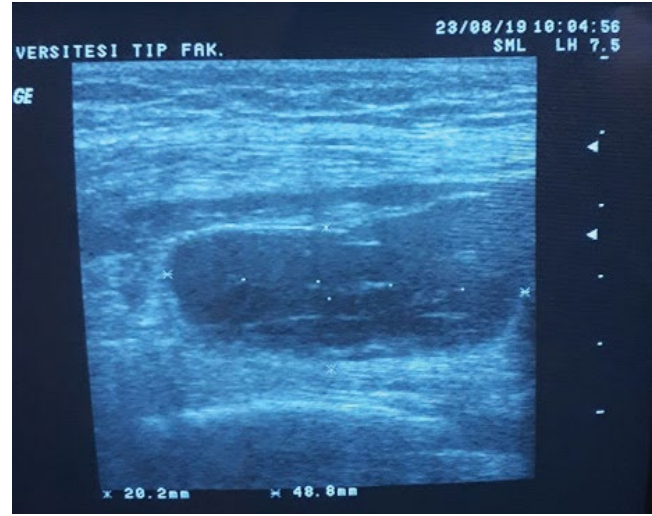

FIGURE 5. Transverse scan $48^{\text {th }}$ hour control ultrasound. Transverse diameter: $20.2 \mathrm{~mm}$

after hip dislocation causing gluteal compartment syndrome [16]. It was observed that the ecchymosis and oedema extended to the sole of the foot and enlarged because of the gravity in the intermuscular haematoma. In contrast, intramuscular haematomas are localized and do not lead to an increase in the ecchymosis area in time [17].

RICE (Rest, Ice, Compression, Elevation) protocol is the first treatment option in muscle traumas. The purpose of RICE Protocol is to stop bleeding that occurs due to injury [18]. Since the patient was questioned, it was determined that he did not apply ice and foot elevation treatment after the trauma. Since the first admission of the patient, RICE protocol was initiated, and regression was detected in the bleeding area in the control ultrasonography.

Ultrasonography is a powerful diagnostic method in musculoskeletal pathologies as it allows real-time, high-resolution examination [19]. The use of ultrasound in emergency services provides practical and reliable data [20]. The limits of the haematoma area were clearly demonstrated with the bedside ultrasound in our patient. Ultrasonography may also be used in the aspiration of the haematomas for treatment purposes. Especially in haematoma aspirations that develop after sports injuries, the accuracy and superiority of ultrasound have been emphasized in previous studies [21]. As we did not consider that we could aspirate the fresh blood with aspiration because of the presence of organized haematoma and fibrin on ultrasound, an invasive procedure was not carried out to the patient. In the acute phase of traumas, ultrasound-guided aspiration of the haematoma and following initiation of the RICE protocol were found as important in terms of accelerating the recovery and preventing relevant complications [18]. 
As a result, the development of haematoma after trauma is expected in patients who use coumadin. It is important to use ultrasound at first admission and in the follow-ups of such patients. Applying haematoma drained at first admission together with ultrasound shows that ultrasound has an important place in the treatment. On the other hand, ultrasound is guiding for treatment in organized haematomas. Ultrasound has several advantages like being practical, inexpensive, radiation-safe and providing serial follow-up, which make it preferable especially in patients who are admitted to the emergency departments.

Funding sources: No funding was received. Authors' contribution: Fatih Demir performed the study design, data collection and analysis, and article's drafting; Gülden Kazancı, study design, analysis and article's drafting; and Jacek Smereka, study design, data collection, and article's drafting; and Damian Gorczyca, study design and article's drafting.

Conflict of interest: All authors declare that they have no conflict of interest.

Ethical approval: All procedures performed in studies involving human participants were in accordance with the ethical standards of the institutional and/or national research committee and with the 1964 Helsinki declaration and its later amendments or comparable ethical standards. This article does not contain any studies with animals performed by any of the authors.

\section{REFERENCES}

1. Petrik M, Stambough J, Rothman R. Posttraumatic Gluteal Compartment Syndrome. Clinical Orthopaedics and Related Research. 1988(231): 127-129, doi: 10.1097/00003086-198806000-00017.

2. Barnes MR, Harper WM, Tomson C, et al. Gluteal compartment syndrome following drug overdose. Injury. 1992; 23(4): 274-275, doi: 10.1016/s0020-1383(05)80017-8.

3. Schmalzried T, Eckardt J. Spontaneous Gluteal Artery Rupture Resulting in Compartment Syndrome and Sciatic Neuropathy. Clinical Orthopaedics and Related Research. 1992(275): 253-257, doi: 10.1097/00003086-199202000-00038.

4. Kumar V, Saeed K, Panagopoulos A, et al. Gluteal compartment syndrome following joint arthroplasty under epidural anaesthesia: a report of 4 cases. J Orthop Surg (Hong Kong). 2007; 15(1): 113-117, doi: 10.1177/230949900701500126, indexed in Pubmed: 17429132.

5. Brumback RJ. Traumatic rupture of the superior gluteal artery, without fracture of the pelvis, causing compartment syndrome of the buttock. A case report. The Journal of Bone \& Joint Surgery. 1990; 72(1): 134-137, doi: 10.2106/00004623-199072010-00023.
6. Bakan B, Garipardıç M, Özkan F, et al. Soft Tissue Bleeding in a Patient With Hemophlia A: a case report. Turk J Phys Med Rehab. 2012; 58: 249-251.

7. Smith $A$, Chitre V, Deo H. Acute gluteal compartment syndrome: superior gluteal artery rupture following a low energy injury. BMJ Case Rep. 2012; 2012, doi: 10.1136/bcr-2012-007710, indexed in Pubmed: 23257274.

8. Ellis M, Frank HG. Myositis ossificans traumatica: with special reference to the quadriceps femoris muscle. J Trauma. 1966; 6(6): 724-738, indexed in Pubmed: 5927121.

9. Ma J, Mateer J, Blaivas M. Emergency ultrasound. McGraw-Hill, New York 2007.

10. Gabbett TJ. Incidence, site, and nature of injuries in amateur rugby league over three consecutive seasons. Br J Sports Med. 2000; 34(2): 98-103, doi: 10.1136/bjsm.34.2.98, indexed in Pubmed: 10786864.

11. Demir M, Demir T, Yaylaci S, et al. Spontaneous abdominal hemorrhage due to warfarin treatment. CHRISMED Journal of Health and Research. 2016; 3(4): 298, doi: 10.4103/2348-3334.190571.

12. Uzunget SC, Evrin T, Uzunget SB, et al. Evaluation of activated charcoal and lipid emulsion treatment in model of acute rivaroxaban toxicity. Am J Emerg Med. 2018; 36(8): 1346-1349, doi: 10.1016/j. ajem.2017.12.039, indexed in Pubmed: 29395759.

13. Liu W, Wang D, Ouyang H, et al. Ultrasound Assessment of Muscle Injury Associated with Closed Limb Fracture. Biomed Res Int. 2019; 2019: 9365291, doi: 10.1155/2019/9365291, indexed in Pubmed: 31309121.

14. Sun Fu, Yuan QL, Zhang YG. Large Buttocks Hematoma Caused by Deep Tissue Massage Therapy. Pain Med. 2015; 16(7): 1445-1447, doi: 10.1111/pme.12726, indexed in Pubmed: 26176792.

15. Buselli $P$, Coco V, Notarnicola $A$, et al. Shock waves in the treatment of post-traumatic myositis ossificans. Ultrasound Med Biol. 2010; 36(3): 397-409, doi: 10.1016/j.ultrasmedbio.2009.11.007, indexed in Pubmed: 20133043.

16. Taylor BC, Dimitris C, Tancevski A, et al. Gluteal compartment syndrome and superior gluteal artery injury as a result of simple hip dislocation: a case report. The lowa Orthopaedic Journal. 2011; 31: 181-186.

17. Tol JL, Hamilton B, Best TM. Palpating muscles, massaging the evidence? An editorial relating to 'Terminology and classification of muscle injuries in sport: The Munich consensus statement'. Br J Sports Med. 2013; 47(6): 340-341, doi: 10.1136/bjsports-2012-091849, indexed in Pubmed: 23222191.

18. Thorsson 0 , Lilja $B$, Nilsson $P$, et al. Immediate external compression in the management of an acute muscle injury. Scand J Med Sci Sports. 1997; 7(3): 182-190, indexed in Pubmed: 9200324.

19. Lee JC, Healy J. Sonography of lower limb muscle injury. AJR Am J Roentgenol. 2004; 182(2): 341-351, doi: 10.2214/ajr.182.2.1820341, indexed in Pubmed: 14736659.

20. Situ-LaCasse E, Grieger RW, Crabbe S, et al. Utility of point-of-care musculoskeletal ultrasound in the evaluation of emergency department musculoskeletal pathology. World J Emerg Med. 2018; 9(4): 262-266.

21. Del Cura JL, Zabala R, Corta I. [Ultrasound-guided interventional procedures in the musculoskeletal system]. Radiologia. 2010; 52(6): 525533, doi: 10.1016/j.rx.2010.07.004, indexed in Pubmed: 20951396. 